\title{
Die geographischen Gesellschaften der Schweiz
}

Um die letzte Jahrhundertwende gab es in der Schweiz sicher sechs, vermutlich sieben eigenständige, aktive geographische Gesellschaften (vgl. Tabelle). International dürfte diese Zahl etwa bei 150 bis 160 gelegen haben, womit ungefähr jede 25 . Gesellschaft schweizerisch gewesen wäre - bei einem Bevölkerungsanteil der Schweiz von weniger als einem Hundertstel bezogen auf die Gesamtbevölkerung Europas (inklusive Rußland) und Nordamerikas. Die Mitgliederzahl der schweizerischen geographischen Gesellschaften belief sich geschätzt auf insgesamt etwa 1300 (aktive und Ehrenmitglieder), was nicht ganz einem halben Promille der damaligen Bevölkerung entspricht und damit vermutlich einen internationalen Spitzenwert darstellt. Läßt sich daraus auf besondere geographische Neigungen der Schweizer schließen? Nach der Gründung der geographischen Gesellschaften in Paris (1821), Berlin (1828) und London (1830) wurden weitere Vereinigungen vergleichbarer Art in immer schnellerem Rhythmus ins Leben gerufen; das letzte Viertel des 19. Jahrhunderts verzeichnete einen fast explosionsartigen Zuwachs. 1858 entstand als erste schweizerische und weltweit vierzehnte Gesellschaft die Société de géographie de Genève.

Was waren die Gründe für diesen Boom? Die Schweizer Delegierten am Brüsseler Internationalen Kongreß für Handelsgeographie 1879 notierten diesbezüglich:

«Die Anstrengungen aller Handel und Industrie treibenden Völker concentriren sich gegenwärtig [...] auf das Bestreben, neue und ergiebige Absatzwege zu finden; es organisirt sich ein förmlicher Wettlauf unter den Nationen [...]. Vor Allem gab die schon seit Jahren andauernde Krise den Hauptanstoß dazu; die Anstrengungen früherer Jahre der Prosperität [...] schufen einen Überfluß an Waaren, den die bisherigen Absatzgebiete nicht mehr zu absorbiren fähig waren. Es ist daher nicht zu verwundern, daß die neuen großartigen Entdeckungen, namentlich im Centrum des afrikanischen Continents, eine Aufregung in der industriellen Welt hervorriefen, die [...] die glücklichsten Folgen verspricht. Namentlich hat dadurch das Studium der Geographie in letzter Zeit bei allen Völkern einen wirklich überraschenden Aufschwung genommen; geographische Gesellschaften schossen überall wie Pilze aus dem Boden [...]. Ganz besonders erfreulich ist dabei die Wahrnehmung, daß durch diesen Wetteifer nicht nur das Wissen gefördert wird, sondern daß mit der Bereicherung an Kenntnissen auch zugleich das Gefühl der Solidarität aller civilisirten Völker in der Erstrebung gemeinsamer Ziele unverkennbar zum Durchbruch gelangt.»'
Daß gewichtige wirtschaftliche Motive allgemein bei der Gründung der geographischen Gesellschaften im Spiel waren, mag in der zitierten Quelle allzusehr im Vordergrund stehen (manchmal aber auch zu Unrecht vernachlässigt worden sein); deutlich sind in dieser Hinsicht jedoch die Statuten und Grundsatzerklärungen einzelner Gesellschaften:

«Unter den geographischen Gesellschaften unseres schweizerischen Vaterlandes ragt neben denjenigen von Bern und Genf, welche mehr das theoretisch-geographische Wissen zu fördern suchen, die Ostschweizerische Geographisch-Commercielle Gesellschaft von St. Gallen durch ihre weitausgreifende und für den schweizerischen Export hochbedeutsame Thätigkeit hervor. Nicht nur daß sie der reichen Industrie der Ostschweiz fort und fort neue Winke über die Cursfähigkeit ihrer Manufakturen zu geben versteht, hat dieselbe [...] auch den nicht $\mathrm{zu}$ ihrem Rayon gehörenden Handelsfirmen der übrigen Schweiz bereitwillige Auskunft über die Verhältnisse ferner Länder ertheilt. Die auswärtigen Correspondenten der Ostschweizerischen Geographisch-Commerciellen Gesellschaft versehen die vaterländisch-kantonale Industriewelt mit den schätzbarsten Fingerzeigen auf günstige Absatzgebiete, die wissenschaftlichen Sammlungen St. Gallens mit den Natur-, Kunst- und Gewerbeprodukten überseeischer Länder und Völker und zugleich die wissenschaftlichen Jahreshefte der Gesellschaft mit vorzüglichen Abhandlungen.

Die Mittelschweiz, vorab Solothurn und Aargau, auch Luzern und Baselland, beherbergen nun eine Exportindustrie, die sich zwar nicht in allen Theilen mit der St. Gallischen messen, wohl aber vergleichen kann. Die aargauische und solothurnische Industrie zählt eine Reihe von Firmen, die ihre Interessen über den ganzen Erdball hin wahrzunehmen wissen. Wollen dieselben sich zu Gunsten ihrer eigenen Waaren oder zu Handen ihrer Reisenden Raths erholen, so sind sie bis jetzt genöthigt, sich nach außen zu wenden.

Unsere Mittelschweiz besitzt aber in der Fremde eine so große Anzahl tüchtig gebildeter Handelsreisender und Industrieller, daß es uns im wohlverstandenen Interesse

Peter Jud, Dr. phil. II, Eidmattstraße 49, 8032 Zürich 
zu liegen scheint, wenn wir selbst eine GeographischCommercielle Gesellschaft gründen. [...].

Die Aufgabe, die sich die correspondirenden Mitglieder unserer Gesellschaft zum Wohle des Vaterlandes auferlegen würden, bestände vorzugsweise in der Einlieferung von Berichten über die Bodenbeschaffenheit, Pflanzen und Thiere, Produktionsweisen und Gewerbserzeugnisse, kurzum über das gesammte Natur- und Geistesleben derjenigen außerschweizerischen Länder und Völker, in welchen sie ihre Thätigkeit entfalten. Von besonderm Werthe aber wäre es, wenn sie uns je und je benachrichtigen würden, welche Produkte unserer Industrie auf ihrem Wirkungsterrain in gegebenen Momenten lohnenden Absatz fänden, welche Boden- und Industrieerzeugnisse ihrer Gegend auch bei uns producirt werden könnten; wenn sie uns endlich durch unmittelbare Zusendung der Boden- und Gewerbsprodukte, der Kunstwerke und Alterthümer, der photographischen Ansichten und Volkstypen ihres Landes in Stand setzen würden, eine Sammlung anzulegen, die sich zu einem Museum zu erweitern und sowohl der Schule, als allen jungen Kaufleuten und Industriellen zum Zwecke des Anschauungsunterrichtes vorzügliche Dienste zu leisten verspräche.. ${ }^{2}$
Namhafte Aargauer Kaufleute und Unternehmer haben im Mai 1884 diesen Aufruf zur Gründung der Mittelschweizerischen Geographisch-Commerciellen Gesellschaft unterzeichnet, so u. a. die Fabrikanten C. F. Bally und H. Zschokke sowie der Verlagsbuchhändler R. Sauerländer. Die Ziele waren also hochgesteckt - entsprechend groß wahrscheinlich schon bald die finanzielle Enttäuschung, weil sich die Vereinsaktivitäten nicht so schnell in den angepeilten Umsatzzuwachs umsetzen lieBen. Die Gesellschaft, die so ambitiös begonnen hatte, überlebte nur rund zwanzig Jahre (vgl. Tabelle). Es wäre sicher lohnend, ihrer Geschichte, die bis jetzt noch nicht aufgearbeitet worden ist, genauer nachzugehen, ebenso wie der Geschichte der Geographischen Gesellschaft Herisau, von der vorläufig kaum noch Spuren sichtbar sind. Diese letztere Vereinigung dürfte sich um den nachmaligen Geographiedozenten der Eidgenössischen Technischen Hochschule, Jakob Früh (1852-1938), gruppiert haben.

Es ist wohl kein Zufall, daß von den geographischen Gesellschaften aus der Gründungsphase nur diejenigen bis heute überlebt haben, die sich an ein Universitätsinstitut anlehnen konnten. Bei diesen Gesellschaften steht der

Tab.: Schweizerische Geographische Gesellschaften

\section{Gründungsjahr Name}

1858

1873

1878

1881-1989 (?) Schweizerische Geographische Gesellschaft

1881-1890 (?) Geographische Gesellschaft Herisau

1884-1905 (?) Mittelschweizerische Geographisch-

1885

$1888-1899$

$1897-1899$

*1899 vereinigt

1911

1923

1946

1951

1967

1969

1989 (?) Commercielle Gesellschaft in Aarau

Société de géographie de Genève

Geographische Gesellschaft Bern

Ostschweizerische Geographisch-Commercielle

Gesellschaft (heute: Ostschweizerische

Société neuchâteloise de géographie

Ethnographische Gesellschaft Zürich*

Geographische Gesellschaft Zürich*

Geographisch-Ethnographische Gesellschaft

Zürich

Verein Schweizerischer Geographielehrer

Geographisch-Ethnologische Gesellschaft Basel

Schweizerische Geomorphologische Gesellschaft -

Société vaudoise de géographie

Schweizerische Gesellschaft

für Angewandte Geographie

Schweizerische Gesellschaft für Kartographie

Verband der Schweizer Geographen

Association suisse de géographie (ASG)

\section{Publikationsorgan}

Le Globe

bis 1972: Jahresbericht; später: Jahrbuch

bis 1945: Jahresbericht, Mitteilungen;

später: Publikationen (neue Folge)

ab 1946: Geographica Helvetica

?

Fernschau

Bulletin

-

bis 1917: Jahresbericht; bis 1945: Mitteilungen; später: Geographica Helvetica

1923-1945: Der Schweizer Geograph; später: Mitteilungen

Mitteilungen; seit 1959: Regio Basiliensis

\section{$-$}

Kartographische Nachrichten

Geographica Helvetica 


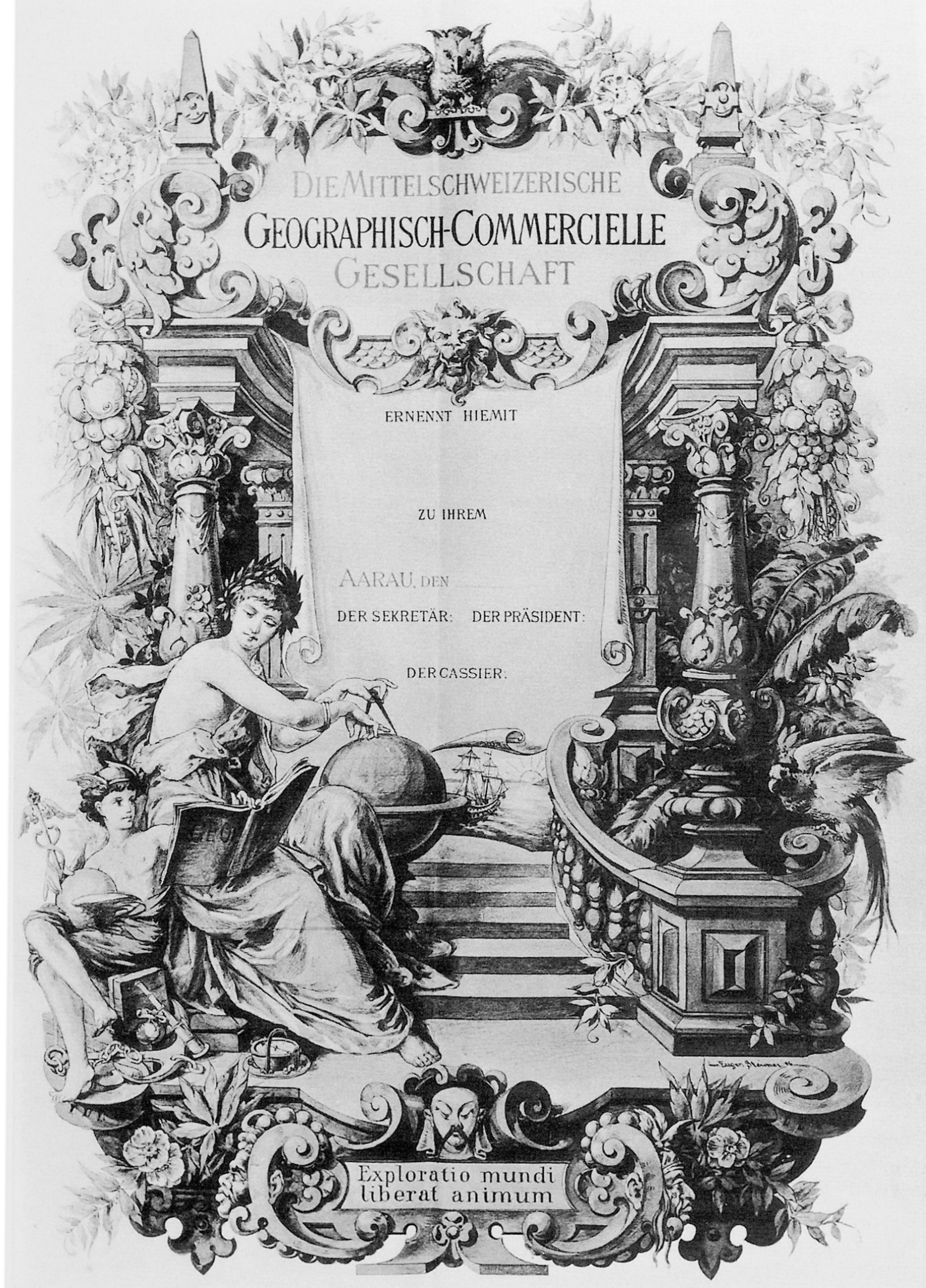

Mitgliederurkunde der Mittelschweizerischen Geographisch-Commerciellen Gesellschaft in Aarau (um 1890). Staatsarchiv des Kantons Aargau, Aarau. Abteilung Nachlässe B. 
oben erwähnte "theoretisch-geographische» Aspekt im Vordergrund. In ihrer ursprünglichen Funktion als Hüterinnen des Wissens und als Zentren der Forschung wurden die geographischen Gesellschaften von den Hochschulinstituten abgelöst; die Rolle der traditionsreichen privaten Vereine hat sich im Laufe der Zeit notgedrungen verändert. ${ }^{3}$ Im Gegensatz zu den jüngeren Gesellschaften fachspezifisch-professionellen Zuschnitts weisen die älteren Gesellschaften nach wie vor den beabsichtigten Laiencharakter auf.

Die Liste der schweizerischen geographischen Gesellschaften wurde im neuen Schweizer Lexikon ${ }^{4}$ nicht ganz vollständig abgedruckt.

Ich bin für Berichtigungen und weiterführende Hinweise dankbar.
Quellen:

' Amrein, K. C., und Künzle-Steger:Bericht über die Verhandlungen des Congrès International de Géographie Commerciale de Bruxelles vom 27. September bis 1. Oktober 1879. In: Jahresbericht der Ostschweizerischen Geographisch-Commerciellen Gesellschaft pro 1879. St. Gallen, 1879.

${ }^{2}$ Die Mittelschweizerische Geographisch-Commercielle Gesellschaft in Aarau [Aufruf zur Gründung und zum Beitritt]; datiert Mai 1884. Staatsarchiv des Kantons Aargau, Aarau. Abteilung Nachlässe $B$.

${ }^{3}$ vgl. dazu: Jud, P.: 100 Jahre Geographisch-Ethnographische Gesellschaft Zürich. In: Geographica Helvetica, 33. Jg. (1989), Nr. 3.

${ }^{4}$ Schweizer Lexikon in sechs Bänden. Bd. 3 (Gen-Kla): "Geographische Gesellschaften". Luzern: Schweizer Lexikon, 1992.

\section{Berichte}

\section{JAHRESTAGUNG 1995}

der Schweizerischen Akademie der Naturwissenschaften SANW

In den Räumen der Hochschule St.Gallen findet vom 6. - 9. September 1995 die SANW Jahrestagung statt, die von der St. Gallischen Naturwissenschaftlichen Gesellschaft NWG organisiert wird. Im Rahmen der Eröffnungsfeier, des Hauptsymposiums und des Forums wird von einer Reihe international anerkannter Fachleute aus Natur- und Wirtschaftswissenschaft das Jahresthema "Naturwissenschaftliche Forschung im Spannungsfeld von Oekologie und Oekonomie" aus den verschiedensten Blickwinkeln zur Diskussion gestellt und daraus Visionen und Botschaften der Wissenschaft an Wirtschschaft und Gesellschaft vermittelt. Als Hauptreferenten konnten gewonnen werden: Prof. Dr. F. Blankart, Staatssekretär BAWI; Prof. Dr. Richard Ernst, ETHZ; Prof. Dr. H.-Ch. Binswanger, HSG; Prof. Dr. J. Tarradellas, EPFL; Prof. Dr. Ch. Wandrey, Forschungszentrum Jülich, und Prof. Dr. H. Plachter, Uni Marburg.
Eine Reihe von Fachgesellschaften und Kommissionen der SANW werden daneben in ihren Veranstaltungen ihren Mitgliedern und vor allem auch Nachwuchskräften die willkommene Gelegenheit bieten, ihre Forschungsergebnisse in Referaten oder auf Posters zu präsentieren.

Die Tatsache, dass sich die schweizerischen $\mathrm{Na}$ turwissenschafter zu ihrer Jahrestagung an der Wirtschaftshochschule der Schweiz treffen, bietet dabei eine augezeichnete Basis für einen interdisziplinären Gedankenaustausch und zur klaren Aufforderung, die Umweltprobleme und die Massnahmen zur Erhaltung unseres Lebensraums weder rein naturwissenschaftlich noch rein ökonomisch bewältigen zu wollen, sondern die Erkenntnisse und Instrumente aller wissenschaftlichen $\mathrm{Be}$ reiche vereint und koordiniert für eine nachhaltige Entwicklung einzusetzen.

Nähere Auskünfte erhalten Sie durch:

\section{SANW 95 Sekretariat}

c/o Grundbauberatung-Geoconsulting AG

Frau Ritter, Helvetiastr. 41

9000 St.Gallen

Tel. 071/ 248844 Telefax 071/248816 